\title{
BMJ Open Association between mid-upper arm circumference and cardiometabolic risk in Chinese population: a cross- sectional study
}

\author{
Yanan Hou, Xu Jia, Liping Xuan, Wen Zhu, Chanjuan Deng, Long Wang, \\ Zhiyun Zhao, Mian Li, Jieli Lu, Yu Xu, Yuhong Chen, Weiqing Wang, Yufang Bi, \\ Min Xu, Tiange Wang
}

To cite: Hou Y, Jia X, Xuan L, et al. Association between mid-upper arm circumference and cardiometabolic risk in Chinese population: a crosssectional study. BMJ Open 2019;9:e028904. doi:10.1136/ bmjopen-2019-028904

- Prepublication history and additional material for this paper are available online. To view these files, please visit the journal online (http://dx.doi org/10.1136/bmjopen-2019028904).

Received 02 January 2019 Revised 04 July 2019 Accepted 19 August 2019

\section{Check for updates}

C Author(s) (or their employer(s)) 2019. Re-use permitted under CC BY-NC. No commercial re-use. See rights and permissions. Published by BMJ.

Shanghai National Clinical Research Center for Endocrine and Metabolic Diseases, Key Laboratory for Endocrine and Metabolic Diseases of the National Health Commission of the PR China, Shanghai Institute of Endocrine and Metabolic Diseases, Ruijin Hospital, Shanghai Jiaotong University School of Medicine, Shanghai, China

Correspondence to Dr Tiange Wang; wtg@live.cn

\section{ABSTRACT}

Objectives Upper body fat has been associated with an unfavourable cardiometabolic risk. We aimed to investigate the associations between mid-upper arm circumference (MUAC), a novel indicator of upper body fat, and a wide spectrum of cardiometabolic risk profiles in Chinese population.

Design and setting Cross-sectional analyses were performed using data from a well-defined community in 2014, Shanghai, China.

Participants A total of 6287 Chinese adults (2310 men and 3977 women) aged 40 years or older.

Outcome measures Multivariable logistic regression model was used to examine the associations of MUAC with cardiometabolic disorders including central obesity, diabetes, hypertension, hypertriglyceridaemia, low highdensity lipoprotein (HDL) cholesterol and subclinical atherosclerosis.

Results In the overall participants, after multivariable adjustment, each $1 \mathrm{SD}(3.13 \mathrm{~cm})$ increment in MUAC was positively associated with central obesity (OR 2.05; $95 \% \mathrm{Cl}$ 1.85 to 2.28), hypertension ( $\mathrm{OR} 1.10 ; 95 \% \mathrm{Cl} 1.03$ to 1.19) and low HDL cholesterol (OR 1.10; $95 \% \mathrm{Cl} 1.01$ to 1.22$)$. Multivariable-adjusted ORs for subclinical atherosclerosis were gradually increased across increasing quartiles of MUAC with the lowest quartile as reference (quartile 2: OR 1.31; $95 \% \mathrm{Cl} 1.09$ to 1.58 ; quartile 3: OR 1.33; $95 \% \mathrm{Cl} 1.10$ to 1.62 ; quartile 4: OR $1.45 ; 95 \% \mathrm{Cl} 1.16$ to $1.80 ; p$ for trend=0.005). Similar but more prominent associations were observed among women than men. In addition, MUAC was significantly interacted with diabetes ( $p$ for interaction $=0.04$ ) and insulin resistance $(p$ for interaction $=0.01$ ) on subclinical atherosclerosis.

Conclusion A greater MUAC was positively associated with higher risks of several cardiometabolic disorders and subclinical atherosclerosis in Chinese adults.

\section{INTRODUCTION}

Cardiometabolic disorders describe a spectrum of interconnected pathological alterations in the cardiovascular system and metabolic organs that symbiotically increase the risk of cardiovascular disease (CVD), which is a major cause of mortality and
Strengths and limitations of this study

- The strengths of this study included a well-defined community setting, a fair sized sample size and comprehensive measurements of cardiometabolic risk profiles.

- The thorough analyses of the associations between mid-upper arm circumference (MUAC) and a wide spectrum of cardiometabolic risk profiles including central obesity, diabetes, hypertension, hypertriglyceridaemia, low high-density lipoprotein cholesterol and subclinical atherosclerosis.

- Although our findings support that MUAC could be a reliable surrogate of upper body adiposity, MUAC is a measurement which reflects both adipose and lean tissue rather than a direct indicator for adiposity.

- Age-related changes in body composition might influence these findings.

- This study was restricted to middle-aged and elderly Chinese adults, and the generalisability of our findings should be cautious to other demographic and ethnic populations.

increasing burden of healthcare expenditure worldwide. $^{1-5}$ Several important cardiometabolic disorders, including obesity, diabetes, insulin resistance, dyslipidaemia and hypertension, are important risk factors for CVD and could be served as targets for early identification and personalised prevention for CVD. ${ }^{2-5}$ In addition, as a common contributor of CVD, atherosclerosis goes through a protracted subclinical phase and could only be detected at an advanced stage of CVD. ${ }^{67}$ Thus, identification of subclinical atherosclerosis in the asymptomatic period is also critical for the prevention of CVD progression. Non-invasive ultrasonography measured carotid intima-media thickness (CIMT) is a well-established clinical index for early arteriosclerosis detection and therefore 
has been extensively adopted to measure subclinical atherosclerosis. $^{8}$

Fat distribution, specifically upper body and visceral adiposity, has been proven highly relevant to cardiovascular risk. ${ }^{910}$ In addition to the conventional body fat indices such as body mass index (BMI) and waist circumference (WC), mid-upper arm circumference (MUAC), a novel anthropometric measurement, has been widely used in the screening of malnutrition, adiposity and chronic diseases. ${ }^{11}$ However, current epidemiological studies have revealed inconsistent results with regard to the association between MUAC and cardiometabolic risk. A cross-sectional study using data from the National Health and Nutrition Examination Survey 1999-2006 circles has reported a positive association between MUAC and insulin resistance in non-obese individuals but no significant association in obese individuals. ${ }^{9}$ In a prospective cohort study of 1061 European elderly participants with a follow-up of approximately 6 years, a larger MUAC was associated with elevated risks of all-cause or CVD mortality. ${ }^{12}$ By contrast, in the Canada Fitness Survey of 10638 adults, a larger MUAC was independently associated with a lower risk of all-cause mortality. ${ }^{13}$ And such inverse association between a larger MUAC and a lower risk of mortality was also documented in the British National Diet and Nutrition Survey of 1054 participants with more than 15 years of follow-up. ${ }^{14}$

So far, most of the previous studies were conducted in European population. Chinese population tends to have a higher percentage of body fat, a weaker willingness on body build and less muscle mass as well as connective tissue as compared with their European counterparts. ${ }^{15}$ These different features in body composition may translate into varying susceptibilities to adiposity-related cardiometabolic disorders. However, comprehensive analyses on associations between MUAC and cardiovascular risk profiles in Chinese population are still limited. Therefore, this study aimed to investigate the association between MUAC and multiple cardiometabolic disorders as well as subclinical atherosclerosis in Chinese population.

\section{METHODS}

\section{Study population}

This is a cross-sectional analysis based on one of the follow-up circles of the established community-based cohort. ${ }^{16}{ }^{17}$ Eligible participants aged 40 years or older were identified from the local residence registration records. There was no restriction on ethnicity or gender. Each eligible participant was recruited by trained community staff and local health workers using a door-todoor invitation method. Participants who consented for the study and signed informed consent were scheduled for health examinations. Briefly, a total of 6570 participants aged 40 years or older were enrolled from Jiading district, Shanghai, China, from August 2014 to May 2015. All participants received anthropometric measurements including height, weight, WC and MUAC, a standard
$75 \mathrm{~g}$ oral glucose tolerance test (OGTT) and a standard questionnaire to collect information regarding social demographic characteristics, education attainment, lifestyle factors and history of disease and medicine. Blood samples were collected for biochemical measurements. In the present study, 283 participants were excluded due to missing data on MUAC or CIMT, and a total of 6287 participants were included in the final analysis. Written informed consents were obtained from all study participants.

\section{Data collection and biochemical measurements}

Detailed information on sociodemographic characteristics, education attainment and lifestyle factors, including smoking and alcohol drinking habits and physical activity, family history and medical history was obtained by using a standard questionnaire administered by trained personnel. Current smokers or alcohol drinkers were defined as persons who consumed any kinds of cigarettes or alcohol regularly in the past 6 months, respectively. Physical activity in terms of metabolic equivalent hour/ week was calculated according to the short form of the International Physical Activity Questionnaire including physical activities both during leisure time and at work. ${ }^{18}$

Anthropometric measurements including height, weight, WC and MUAC were assessed by well-trained physician according to a standard protocol. Body height and weight were measured with participants wearing light clothes without shoes to the nearest $0.1 \mathrm{~cm}$ and $0.1 \mathrm{~kg}$, respectively. BMI was calculated as body weight in kilograms divided by body height in metres squared $(\mathrm{kg} /$ $\mathrm{m}^{2}$ ). MUAC was measured on the upper left arm (halfway between the acromion process and the olecranon process) with the participants' bilateral arms hanging down naturally. WC was measured at the level of the umbilicus with the participants in the standing position. Systolic and diastolic blood pressures were measured in the non-dominant arm with an automated electronic sphygmomanometer (OMRON Model HEM-752 FUZZY, Omron, Dalian, China) three times (averaged for analysis) consecutively with $1 \mathrm{~min}$ interval after at least 5 min rest in a seated position.

All participants were undertaken a $75 \mathrm{~g}$ OGTT after an overnight fasting. Fasting plasma glucose and OGTT 2-hour plasma glucose were measured using hexokinase method on a clinical chemistry diagnostic system (Modular P800; Roche, Basel, Switzerland). Serum concentrations of total cholesterol, triglycerides, high-density lipoprotein (HDL) cholesterol and low-density lipoprotein (LDL) cholesterol were measured by an autoanalyser (Modular E170; Roche). High-sensitive C-reactive protein concentration was determined by highly sensitive competitive immunoassay (antigens and antibodies from Beckman coulter IMMAGE800, America). Serum insulin was measured by using the electrochemiluminescence methods on an immunology analyser (RIABEAD II; Abbott, Tokyo, Japan). Homeostasis model assessment of insulin resistance (HOMA-IR) was 
calculated as fasting insulin $(\mu \mathrm{IU} / \mathrm{mL}) \times$ fasting plasma glucose $(\mathrm{mmol} / \mathrm{L}) / 22.5$, and insulin resistance was defined as HOMA-IR $\geq 2.8$, which is the cut-off point for the highest quartile of the total participants.

\section{Definitions of cardiometabolic risk profile}

Type 2 diabetes was defined as fasting plasma glucose $\geq 7.0 \mathrm{mmol} / \mathrm{L}$, or OGTT 2-hour plasma glucose $\geq 11.1 \mathrm{mmol} / \mathrm{L}$ or use of antidiabetic agents. ${ }^{19}$ Hypertension was defined as systolic blood pressure $\geq 140 \mathrm{~mm} \mathrm{Hg}$, or diastolic blood pressure $\geq 90 \mathrm{~mm} \mathrm{Hg}$ or current taking an antihypertensive medication. Central obesity was defined as WC $\geq 102 \mathrm{~cm}$ for men and $\geq 88 \mathrm{~cm}$ for women. Hypertriglyceridaemia was defined as triglycerides $\geq 2.26 \mathrm{mmol} / \mathrm{L}$. Low HDL cholesterol was defined as HDL cholesterol $<1.04 \mathrm{mmol} / \mathrm{L}$.

CIMT measurement was conducted by a trained sonographer using a high-resolution B-mode tomographic ultrasound system (Esaote Biomedica, Genoa, Italy) with a linear $7.5 \mathrm{MHz}$ transducer. ${ }^{17}$ The position of CIMT measurement was recorded on the far wall of both right and left common carotid arteries, $1.5 \mathrm{~cm}$ proximal to the bifurcation. CIMT was measured on-line at the end of diastole as a distance from the leading edge of the first echogenic line to that of the second. These two lines represent the lumen-intima interface and collagen-contained upper layer of tunic adventitia, respectively. Subclinical atherosclerosis was defined as a bilateral CIMT average greater than $0.8 \mathrm{~mm}$, which is the highest quartile cut-off point of the total participants.

\section{Statistical analyses}

SAS V.9.2 (SAS Institute) was used for statistical analysis. Continuous variables were described as means \pm SDs or medians (IQRs), and categorical variables as numbers (percentages). Variables with skewed distributions, such as HOMA-IR, triglycerides and C-reactive protein, were normalised by logarithmic transformation before analysis.

Participants were divided into four subgroups according to quartiles of MUAC. The ranges of MUAC within each quartile were $15.5-27.1 \mathrm{~cm}$ for quartile $1 ; 27.2-29.1 \mathrm{~cm}$ for quartile 2; $29.2-31.2 \mathrm{~cm}$ for quartile 3 ; and $31.3-43.3 \mathrm{~cm}$

Table 1 Characteristics of study participants according to quartiles of mid-upper arm circumference

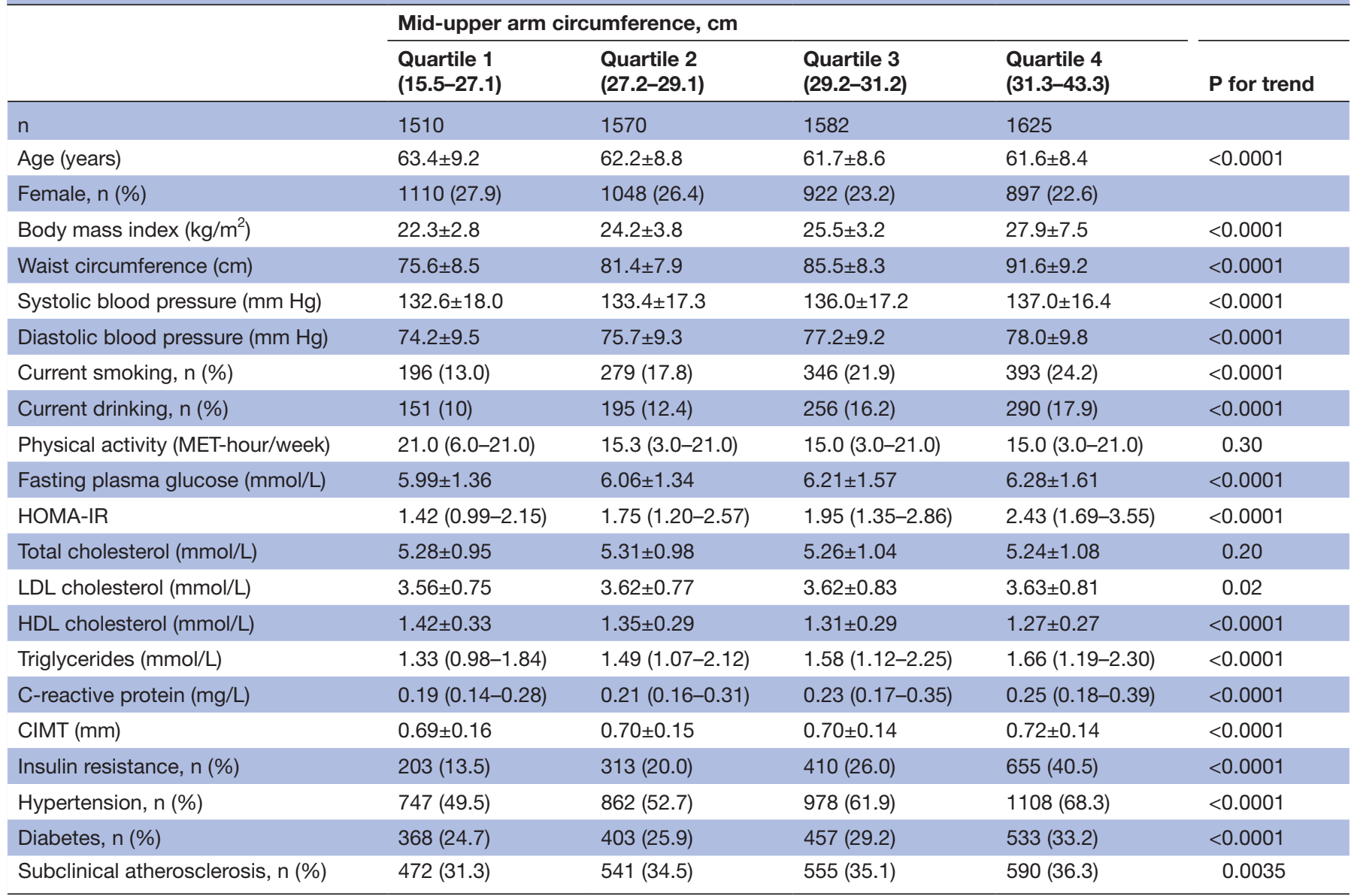

Data are presented as means $\pm \mathrm{SD}$, or medians (IQRs) for skewed variables, or number (proportions) for categorical variables. $\mathrm{P}$ values were calculated from one-way analysis of variance (ANOVA) for continuous variables and $\chi^{2}$ test for categorical variables. Subclinical atherosclerosis was defined as CIMT $\geq 0.8 \mathrm{~mm}$, which is the cut-off point for the highest quartile of the total participants. Insulin resistance was defined as HOMA-IR $\geq 2.8$, which is the cut-off point for the highest quartile of the total participants.

CIMT, carotid intima-media thickness; HDL, high-density lipoprotein; HOMA-IR, homeostasis model assessment of insulin resistance; LDL, lowdensity lipoprotein; MET, metabolic equivalent task. 
Table 2 Association between mid-upper arm circumference and multiple cardiometabolic disorders in total and sex-specific participants

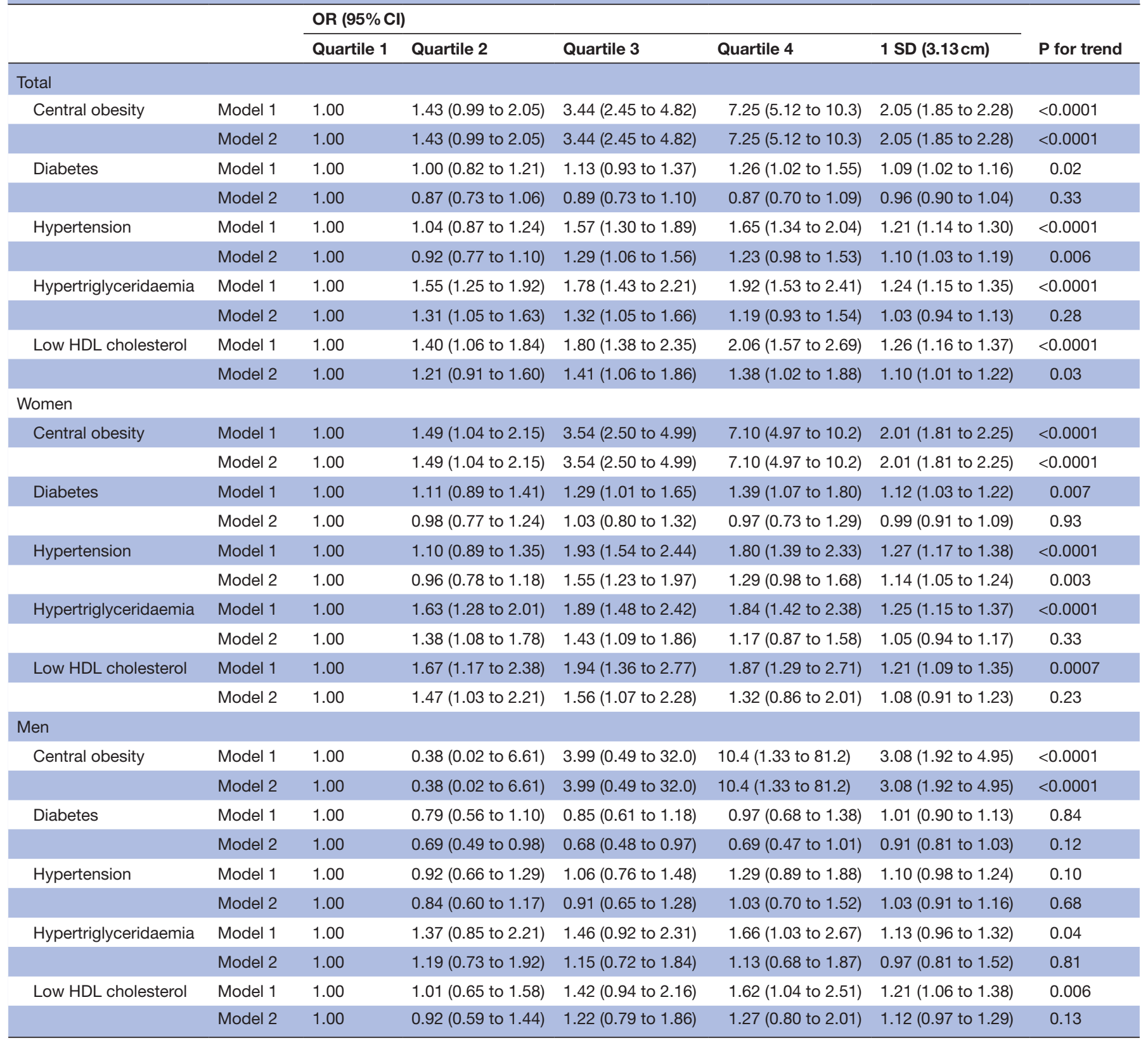

Data were presented as OR and $95 \% \mathrm{Cl}$. P values were calculated from multivariable logistic regression analysis in quartile of mid-upper arm circumference. Model 1: adjusted for age (years), sex, body mass index $\left(\mathrm{kg} / \mathrm{m}^{2}\right)$, current smoking (yes or no), current drinking (yes or no), physical activity (MET-hour/week). Model 2: further adjusted for waist circumference $(\mathrm{cm})$, except for central obesity.

$\mathrm{HDL}$, high-density lipoprotein; MET, metabolic equivalent task.

for quartile 4. Linear regression analysis was used to test the trend of continuous variables across MUAC quartiles and the Cochran-Armitage trend $\chi^{2}$ test was used to test the differences of proportions of categorical variables. Multivariable linear regression and multivariable logistic regression analyses were conducted to assess the associations of MUAC with multiple cardiometabolic disorders and subclinical atherosclerosis, with adjustment for age, sex, BMI, WC, current smoking, current drinking, physical activity, C-reactive protein, total cholesterol, HDL cholesterol, LDL cholesterol, triglycerides, fasting plasma glucose and systolic blood pressure. Stratification analyses by age, BMI, WC, diabetes, insulin resistance and hypertension were also performed. Interactions were tested by adding the respective multiplicative terms in the models simultaneously. Statistical significance was set to a two-sided $\mathrm{p}$ value of less than 0.05 .

\section{Patient and public involvement}

This study was conducted without patient and public involvement. No patients were invited to participate in the development of the research question and outcome 
measures, the study design and the interpretation of the results. The findings from this study will be disseminated to the participants after the results are published in a peer-reviewed journal.

\section{RESULTS}

\section{Characteristics of the study participants}

Study participants included $2310(36.7 \%)$ men and 3977 $(63.3 \%)$ women with an average age of 62.2 years (SD: 8.78). Sociodemographic and clinical characteristics of the study participants according to MUAC quartiles are shown in table 1 . Participants with a large MUAC were younger, had higher levels of BMI, WC, systolic and diastolic blood pressures, fasting plasma glucose, HOMAIR, LDL cholesterol, triglycerides, C-reactive protein and CIMT, had higher proportions of insulin resistance, hypertension, diabetes and subclinical atherosclerosis and had lower levels of HDL cholesterol (all $\mathrm{p}<0.05$; table 1). Consistent linear associations were observed between MUAC and these cardiometabolic profiles (online supplementary table 1 ).

\section{Association between MUAC and cardiometabolic disorders}

As shown in table 2, the multivariable-adjusted OR per 1 SD increment $(3.13 \mathrm{~cm})$ in MUAC was 2.05 (95\% CI 1.85 to 2.28) for central obesity, 1.09 (95\% CI 1.02 to 1.16 ) for diabetes, 1.21 (95\% CI 1.14 to 1.30) for hypertension, 1.24 (95\% CI 1.15 to 1.35 ) for hypertriglyceridaemia and
1.26 (95 CI 1.16 to 1.37) for low HDL cholesterol. Most of these associations were not substantially changed after additional adjustment for WC, except for diabetes and hypertriglyceridaemia. When stratified by sex, similar but more prominent associations between MUAC and these cardiometabolic disorders were observed among women.

\section{Association between MUAC and subclinical atherosclerosis}

In total participants, we observed strong and positive association between MUAC and subclinical atherosclerosis with full adjustment (table 3). The ORs for subclinical atherosclerosis were 1.31 (95\% CI 1.09 to 1.58 ) for quartile 2 of MUAC, 1.33 (95\% CI 1.10 to 1.62) for quartile 3 and 1.45 (95\% CI 1.16 to 1.80 ) for quartile 4 as compared with the lowest quartile ( $\mathrm{p}$ for trend $=0.005$ ). When stratified by sex, statistically significant results were observed among women but not among men.

\section{Stratification analyses by traditional cardiovascular risk factors}

We further examined the association between MUAC and subclinical atherosclerosis across strata of traditional cardiometabolic risk factors. In women, MUAC was associated with subclinical atherosclerosis among participants aged $\geq 62$ years, with BMI $\geq 25 \mathrm{~kg} / \mathrm{m}^{2}$ or $\mathrm{WC}<88 \mathrm{~cm}$ (figure 1A), and among participants with diabetes, insulin resistance, or hypertension (figure 1B). Moreover, there were significant interactions of MUAC with diabetes and insulin resistance on subclinical atherosclerosis ( $p$ for

Table 3 Association between mid-upper arm circumference and subclinical atherosclerosis in total and sex-specific participants

\begin{tabular}{|c|c|c|c|c|c|c|}
\hline & \multicolumn{5}{|c|}{ OR $(95 \% \mathrm{Cl})$} & \multirow[b]{2}{*}{ P for trenc } \\
\hline & Quartile 1 & Quartile 2 & Quartile 3 & Quartile 4 & $1 \mathrm{SD}(3.13 \mathrm{~cm})$ & \\
\hline Total & & & & & & $0.20^{*}$ \\
\hline Model 1 & 1.00 & 1.16 (1.00 to 1.34$)$ & 1.19 (1.02 to 1.38$)$ & 1.25 (1.08 to 1.45$)$ & 1.06 (1.01 to 1.12$)$ & 0.004 \\
\hline Model 2 & 1.00 & 1.30 (1.08 to 1.56$)$ & 1.30 (1.07 to 1.58$)$ & 1.35 (1.09 to 1.67$)$ & 1.06 (0.98 to 1.15$)$ & 0.013 \\
\hline Cases/participants & $287 / 1110$ & $327 / 1048$ & $275 / 922$ & $282 / 897$ & $1171 / 3977$ & \\
\hline Model 1 & 1.00 & 1.30 (1.08 to 1.57$)$ & $1.22(1.00$ to 1.48$)$ & $1.32(1.09$ to 1.60$)$ & 1.07 (1.00 to 1.14$)$ & 0.014 \\
\hline Model 2 & 1.00 & 1.54 (1.24 to 1.93$)$ & 1.43 (1.18 to 1.82$)$ & 1.53 (1.17 to 2.02$)$ & 1.11 (1.01 to 1.23$)$ & 0.007 \\
\hline Model 3 & 1.00 & 1.54 (1.24 to 1.93$)$ & 1.42 (1.11 to 1.83$)$ & 1.66 (1.26 to 2.20$)$ & 1.14 (1.03 to 1.26$)$ & 0.002 \\
\hline Model 2 & 1.00 & 0.89 (0.64 to 1.23$)$ & $1.04(0.75$ to 1.44$)$ & $1.00(0.70$ to 1.45$)$ & 0.97 (0.85 to 1.11$)$ & 0.71 \\
\hline Model 3 & 1.00 & $0.90(0.65$ to 1.25$)$ & 1.06 (0.76 to 1.48$)$ & $1.05(0.72$ to 1.52$)$ & 0.99 (0.86 to 1.06$)$ & 0.54 \\
\hline
\end{tabular}

Data were presented as $\mathrm{OR}$ and $95 \% \mathrm{Cl}$. P values were calculated from multivariable logistic regression analysis.

*The interaction of mid-upper arm circumference (MUAC) with sex on subclinical atherosclerosis. Model 1: unadjusted. Model 2: adjusted for age (years), sex, body mass index $\left(\mathrm{kg} / \mathrm{m}^{2}\right)$, current smoking (yes or no), current drinking (yes or no), physical activity (MET-hour/week), waist circumference $(\mathrm{cm})$. Model 3: further adjusted for C-reactive protein $(\mathrm{mg} / \mathrm{L})$, total cholesterol $(\mathrm{mmol} / \mathrm{L})$, high-density lipoprotein $(\mathrm{HDL})$ cholesterol $(\mathrm{mmol} / \mathrm{L})$, low-density lipoprotein (LDL) cholesterol $(\mathrm{mmol} / \mathrm{L})$, triglycerides $(\mathrm{mmol} / \mathrm{L})$, fasting plasma glucose $(\mathrm{mmol} / \mathrm{L})$ and systolic blood pressure $(\mathrm{mm}$ $\mathrm{Hg})$.

MET, metabolic equivalent task. 


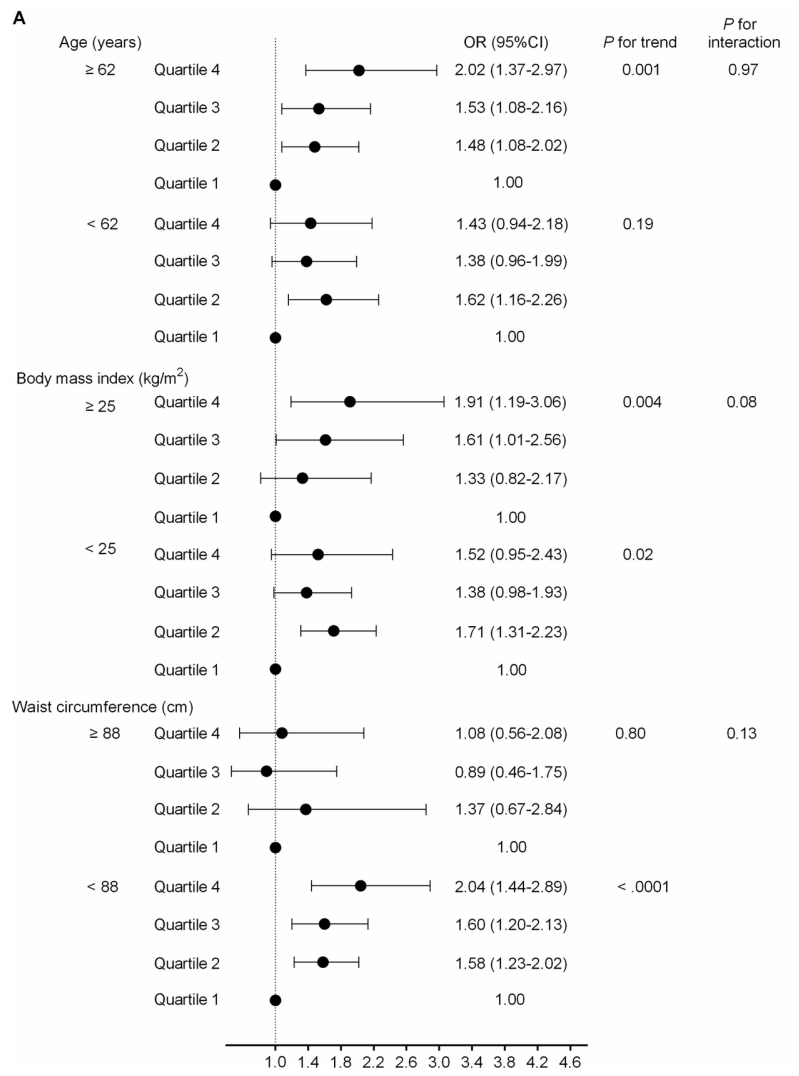

B

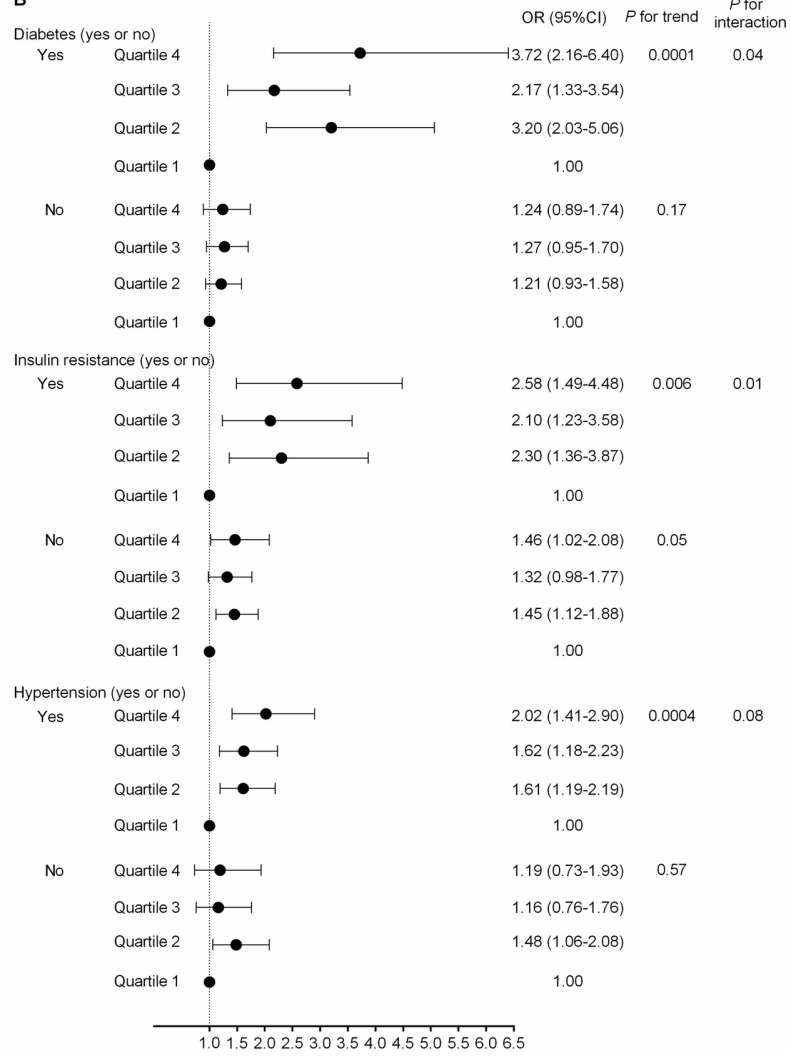

Figure 1 Stratification analysis of the association between mid-upper arm circumference (MUAC) and subclinical atherosclerosis in women. (A) All participants were divided into subgroups based on their average age (age $<62$ years, age $\geq 62$ years), body mass index (BMl $<25 \mathrm{~kg} / \mathrm{m}^{2}$, BMl $\geq 25 \mathrm{~kg} / \mathrm{m}^{2}$ ) or waist circumference $(\mathrm{WC}<88 \mathrm{~cm}$ or WC $\geq 88 \mathrm{~cm}$ ). (B) All participants were divided into subgroups based on diabetes (yes or no), insulin resistance (yes or no) or hypertension (yes or no). Data were presented as $\mathrm{OR}$ and $95 \% \mathrm{Cl}$. P values were calculated from multivariable logistic regression analysis. Adjusted for age (years), body mass index $\left(\mathrm{kg} / \mathrm{m}^{2}\right)$, current smoking (yes or no), current drinking (yes or no), physical activity (MET-hour/week), WC (cm), C-reactive protein $(\mathrm{mg} / \mathrm{L})$, total cholesterol (mmol/L), high-density lipoprotein (HDL) cholesterol (mmol/L), low-density lipoprotein $(\mathrm{LDL})$ cholesterol $(\mathrm{mmol} / \mathrm{L})$, triglycerides $(\mathrm{mmol} / \mathrm{L})$, fasting plasma glucose $(\mathrm{mmol} / \mathrm{L})$ and systolic blood pressure $(\mathrm{mm} \mathrm{Hg}) . \mathrm{MET}$, metabolic equivalent task.

interaction $=0.04$ and 0.01 , respectively). These association patterns were not observed in men (online supplementary figure 1).

To further evaluate whether the association of MUAC with subclinical atherosclerosis is influenced by BMI and $\mathrm{WC}$, we replicated the analyses within combinations of BMI and WC. In women, those with higher WC were more likely to have larger MUAC, regardless of BMI levels (figure 2A). MUAC was also independently associated with subclinical atherosclerosis in the combination of WC $<88 \mathrm{~cm}$ and $\mathrm{BMI}<25 \mathrm{~kg} / \mathrm{m}^{2}$ (MUAC each 1-quartile increment, OR 1.22; $95 \%$ CI 1.05 to 1.43 ) and the combination of $\mathrm{WC}<88 \mathrm{~cm}$ and $\mathrm{BMI} \geq 25 \mathrm{~kg} / \mathrm{m}^{2}$ (MUAC each 1-quartile increment, OR 1.40; 95\% CI 1.11 to 1.77 ) (figure $2 \mathrm{~B}$ ).

\section{DISCUSSION}

In this cross-sectional study of 6287 community-dwelling Chinese adults, MUAC was positively associated with a series of cardiometabolic disorders including central obesity, hypertension, low HDL cholesterol and subclinical atherosclerosis. These associations were independent from traditional cardiovascular risk factors, and were more prominent in women. Moreover, we observed significant interactions of MUAC with diabetes and insulin resistance in relation to subclinical atherosclerosis.

MUAC has been accepted as a widely used indicator of upper body adiposity in children, adolescents and adults. ${ }^{2021}$ Previous studies have shown mixed results on the associations between MUAC and CVD. ${ }^{22-25}$ Findings from a retrospective cohort study of 570 Japanese adults have suggested that MUAC may play a complementary role to $\mathrm{BMI}$ in predicting prognosis in patients with heart failure. ${ }^{22}$ In addition, a cross-sectional study of 93 pubertal obese adolescents from Brazil has associated a larger MUAC with a higher level of HOMA-IR and a higher cardiometabolic risk score. ${ }^{23}$ On the contrary, results from the Health Effects of Arsenic Longitudinal Study of 562 middle-aged participants who were free of CVD in rural Bangladesh have shown no relationship between MUAC and CIMT. ${ }^{24}$ Our study has extended the existing evidence by demonstrating that a greater MUAC was associated with higher prevalent risk of multiple cardiometabolic disorders including central obesity, hypertension, low HDL cholesterol and subclinical atherosclerosis 

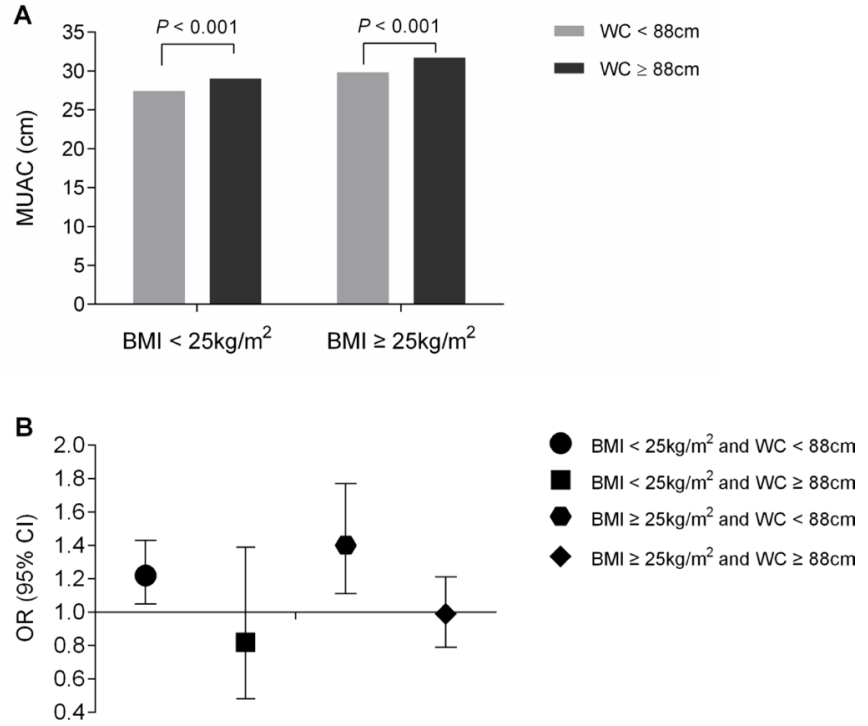

Figure 2 Association between MUAC and subclinical atherosclerosis according to combined categories of $\mathrm{BMI}$ and WC. (A) MUAC in the BMI and WC subgroups in women. (B) Association of MUAC with risk of subclinical atherosclerosis in the BMI and WC subgroups in women. Adjusted for age (years), body mass index $\left(\mathrm{kg} / \mathrm{m}^{2}\right)$, current smoking (yes or no), current drinking (yes or no), physical activity (MET-hour/ week), WC (cm), C-reactive protein ( $\mathrm{mg} / \mathrm{L})$, total cholesterol $(\mathrm{mmol} / \mathrm{L})$, high-density lipoprotein (HDL) cholesterol $(\mathrm{mmol} / \mathrm{L})$, low-density lipoprotein (LDL) cholesterol $(\mathrm{mmol} / \mathrm{L})$, triglycerides $(\mathrm{mmol} / \mathrm{L})$, fasting plasma glucose $(\mathrm{mmol} / \mathrm{L})$ and systolic blood pressure $(\mathrm{mm} \mathrm{Hg})$. BMI, body mass index; MET, metabolic equivalent task; MUAC, mid-upper arm circumference; WC, waist circumference.

in Chinese adults, particularly among women. Central obesity, hypertension, low HDL cholesterol and subclinical atherosclerosis have been robustly associated with increased risks of CVD, therefore detecting more effective risk factors for these cardiometabolic disorders is critical to the prevention of CVD. Our findings highlight the importance of paying more attention to women with higher MUAC in the early identification and precise prevention of cardiometabolic disorders.

Explanations for the observations between MUAC and cardiometabolic risk are multifactorial. Our study found that MUAC level tended to decrease with age and there was a stronger association between MUAC and subclinical atherosclerosis among women aged 62 years or older. Ageing is a critical factor in the changing process of metabolism and body composition. Fat-free mass and muscle mass reduced while substantial visceral fat increased with ageing, even under the condition of body weight unchanged. ${ }^{25}$ It has been documented that individuals aged greater than 65 years would suffer a reduction in muscle mass over $25 \%$ per year; and this rate can be accelerated to $50 \%$ per year for those older than 80 years. ${ }^{25-27}$ In addition, we found that MUAC was associated with subclinical atherosclerosis in a sex-specific manner. One plausible explanation for the sex difference in MUAC-subclinical atherosclerosis relationship may be due to the biological differences between men and women, such as hormones effect, immune system responses, muscle capacity and physical function. For instance, men tend to have greater muscle capacity and higher muscle mass than women due to higher levels of testosterone. ${ }^{26}$ Body fat redistributes to upper body and to a preferential adiposity around the waist with age and this trend was more obvious in women than in men. ${ }^{27}$ The sex difference in redistribution of body fat may partly contribute to the observed more predominant associations between MUAC and cardiometabolic disorders among women.

The strength of this study is the comprehensive analyses of the association between MUAC and a wide spectrum of cardiometabolic risk profiles in a well-defined community setting with fair sized sample and desirable population homogeneity. Several limitations should be considered. First, due to a cross-sectional nature of the present study, no causal inference can be drawn. Second, although our findings supported that MUAC could be a reliable surrogate of upper body adiposity, MUAC is a measurement which reflects both adipose and lean tissue rather than a direct indicator for adiposity. Third, although we have carefully adjusted for multiple confounders, age-related changes in body composition might influence these findings. Fourth, MUAC measurement was performed on the left arm, though it should be determined on the non-dominant arm. Given that the majority of Chinese population were right-handers, measurement protocol employed in our study for MUAC was acceptable. Finally, this study was restricted to middle-aged and elderly Chinese adults, and the generalisability of our findings should be cautious to other demographic and ethnic populations.

In conclusion, our study provided novel evidence of positive associations between MUAC and cardiometabolic disorders as well as subclinical atherosclerosis in Chinese population. Our findings suggest that MUAC, as a convenient and inexpensive measurable metric, can be potentially used as a risk stratification indicator in the early detection and prevention of CVD.

Acknowledgements We thank all the study participants for their participation and contribution. The present study would not have been possible without the participation of the participants.

Contributors All authors had full access to all of the data in the study and take responsibility for the integrity of the data and the accuracy of the data analysis. Study concept and design: TW, MX, YB and WW. Acquisition of data: all authors. Interpretation of data: $Y H, T W, M X, Y B$ and WW. Drafting of the manuscript: $Y H$. Critical revision of the manuscript: TW, MX, YB and WW. Statistical analysis: YH and XJ.

Funding This work was supported by grants from the National Natural Science Foundation of China (81770842 and 81561128019), the Ministry of Science and Technology of China (2018YFC1311705, 2016YFC1305600 and 2016YFC1304904), the Shanghai Pujiang Program (18PJ1409600), the 'Shanghai Municipal Education Commission-Gaofeng Clinical Medicine Grant Support' from Shanghai Jiao Tong University School of Medicine (20171901), the Shanghai Science and Technology Commission (YDZX20173100004881) and the Shanghai Shen-Kang Hospital Development Center (SHDC12016202).

Competing interests None declared.

Patient consent for publication Not required. 
Ethics approval The study protocol was approved by the Institutional Review Board of Ruijin Hospital Affiliated to Shanghai Jiao Tong University School of Medicine.

Provenance and peer review Not commissioned; externally peer reviewed.

Data availability statement All data relevant to the study are included in the article or uploaded as supplementary information.

Open access This is an open access article distributed in accordance with the Creative Commons Attribution Non Commercial (CC BY-NC 4.0) license, which permits others to distribute, remix, adapt, build upon this work non-commercially, and license their derivative works on different terms, provided the original work is properly cited, appropriate credit is given, any changes made indicated, and the use is non-commercial. See: http://creativecommons.org/licenses/by-nc/4.0/.

\section{REFERENCES}

1. Hertle E, Stehouwer CDA, van Greevenbroek MMJ. The complement system in human cardiometabolic disease. Mol Immunol 2014;61:135-48.

2. Kaur J. A comprehensive review on metabolic syndrome. Cardiol Res Pract 2014;2014:1-21.

3. WHO. Cardiovascular diseases (CVDs), 2015. Available: www.who. int/mediacentre/factsheets/fs317/en/index.html [Accessed 15 May 2016].

4. Leal J, Luengo-Fernández R, Gray A, et al. Economic burden of cardiovascular diseases in the enlarged European Union. Eur Heart $J$ 2006;27:1610-9.

5. Mozaffarian D, Benjamin EJ, Go AS, et al. Heart disease and stroke statistics--2015 update: a report from the American Heart Association. Circulation 2015;131:e29-322.

6. Mao Z, Wu F, Shan Y. Identification of key genes and miRNAs associated with carotid atherosclerosis based on mRNA-seq data. Medicine 2018;97:e9832.

7. Fernández-Friera L, Peñalvo JL, Fernández-Ortiz A, et al. Prevalence, vascular distribution, and multiterritorial extent of subclinical atherosclerosis in a middle-aged cohort: the PESA (progression of early subclinical atherosclerosis) study. Circulation 2015;131:2104-13.

8. Lorenz MW, Markus HS, Bots ML, et al. Prediction of clinical cardiovascular events with carotid intima-media thickness: a systematic review and meta-analysis. Circulation 2007;115:459-67.

9. Chao Y-P, Lai Y-F, Kao T-W, et al. Mid-arm muscle circumference as a surrogate in predicting insulin resistance in non-obese elderly individuals. Oncotarget 2017;8:79775-84.

10. Nielsen S, Guo Z, Johnson CM, et al. Splanchnic lipolysis in human obesity. J Clin Invest 2004;113:1582-8.

11. Ho S-C, Wang J-Y, Kuo H-P, et al. Mid-arm and calf circumferences are stronger mortality predictors than body mass index for patients with chronic obstructive pulmonary disease. Int J Chron Obstruct Pulmon Dis 2016;11:2075-80.
12. Ellen L, Wanda J, Lisette C. Associations between changes in anthropometric measures and mortality in old age: a role for midupper arm circumference? JAMDA 2013;187:e193.

13. Mason C, Craig CL, Katzmarzyk PT. Influence of central and extremity circumferences on all-cause mortality in men and women. Obesity 2008;16:2690-5.

14. Bates CJ, Hamer M, Mishra GD. A study of relationships between bone-related vitamins and minerals, related risk markers, and subsequent mortality in older British people: the National Diet and Nutrition Survey of People Aged 65 Years and Over. Osteoporos Int 2012;23:457-66.

15. Wang $T$, Zhao $Z, X u Y$, et al. Insulin resistance and $\beta$-cell dysfunction in relation to cardiometabolic risk patterns. J Clin Endocrinol Metab 2018;103:2207-15.

16. Huang $\mathrm{Y}, \mathrm{Bi} \mathrm{Y}, \mathrm{Xu} \mathrm{M}$, et al. Nonalcoholic fatty liver disease is associated with atherosclerosis in middle-aged and elderly Chinese. Arterioscler Thromb Vasc Biol 2012;32:2321-6.

17. Chen $\mathrm{Y}, \mathrm{Xu} \mathrm{M}$, Wang $\mathrm{T}$, et al. Advanced fibrosis associates with atherosclerosis in subjects with nonalcoholic fatty liver disease. Atherosclerosis 2015;241:145-50.

18. Craig CL, Marshall AL, Sjöström M, et al. International physical activity questionnaire: 12-country reliability and validity. Med Sci Sports Exerc 2003;35:1381-95.

19. Alberti KG, Zimmet PZ, Definition ZPZ. Definition, diagnosis and classification of diabetes mellitus and its complications. Part 1: diagnosis and classification of diabetes mellitus provisional report of a who consultation. Diabet Med 1998;15:539-53.

20. Mramba L, Ngari M, Mwangome M, et al. A growth reference for mid upper arm circumference for age among school age children and adolescents, and validation for mortality: growth curve construction and longitudinal cohort study. BMJ 2017;358:j3423.

21. Wu L-W, Lin Y-Y, Kao T-W, et al. Mid-Arm circumference and allcause, cardiovascular, and cancer mortality among obese and nonobese us adults: the National health and nutrition examination survey III. Sci Rep 2017;7:2302.

22. Kamiya K, Masuda T, Matsue Y, et al. Complementary role of arm circumference to body mass index in risk stratification in heart failure. JACC Heart Fail 2016;4:265-73.

23. Araújo AJS, Santos ACO, Prado WL. Body composition of obese adolescents: association between adiposity indicators and cardiometabolic risk factors. J Hum Nutr Diet 2017;30:193-202.

24. Ge W, Parvez F, Wu F, et al. Association between anthropometric measures of obesity and subclinical atherosclerosis in Bangladesh. Atherosclerosis 2014;232:234-41.

25. Tom SE, Cooper R, Patel KV, et al. Menopausal characteristics and physical functioning in older adulthood in the NHANES III. Menopause 2012;19.

26. Haizlip KM, Harrison BC, Leinwand LA, et al. Sex-based differences in skeletal muscle kinetics and fiber-type composition. Physiology 2015;30:30-9.

27. Wells JCK, Treleaven P, Cole TJ. Bmi compared with 3-dimensional body shape: the UK national sizing survey. Am J Clin Nutr 2007;85:419-25. 